\title{
Assessing Implicit and Explicit Grammatical Knowledge
}

\author{
Gordana Bojicic \\ Facoltà di Filosofia di Niksic, Università del Montenegro \\ bgole81@yahoo.com
}

\section{Doi:10.5901/jesr.2014.v4n4p251}

\begin{abstract}
The study of grammar has always had an important role in the history of foreign language teaching. Today, grammatical competence is seen as an integral part of communicative ability, which represents the aim of language learning and teaching process. The aim of this paper is to offer the most important theoretical views related to the grammatical competence and implicit and explicit grammatical knowledge. We will also try to establish grammatical descriptors based on the most frequent grammatical structures contained in the curriculum for Italian language in Montenegrin elementary schools at level A2. In order to achieve the goals we have set, we have tested 547 ninth grade students of 25 Montenegrin schools. The aim of the testing was to determine the extent to which the tested students fulfilled the requirements listed in the curriculum for Italian language. We have also determined their implicit and explicit grammatical knowledge. After the discussion of the results of the grammar test, we will try to find how the students' achievement on grammatical tests can be improved.
\end{abstract}

Keywords: grammar, explicit, grammatical competence, grammatical knowledge, implicit, Italian language, test.

\section{Introduzione}

Sul ruolo della grammatica nell'ambito dell'insegnamento di una LS si sono avvicendate diverse opinioni che, come dimostrano i recenti studi nell'ambito della linguistica acquisizionale, hanno sempre sottolineato il fatto che la competenza grammaticale rappresenti un fattore importante nel processo dell'apprendimento di una LS. Infatti, senza un certo repertorio di conoscenze grammaticali ed un adeguato livello di competenza, non ci si può aspettare che lo studente sia in grado di comunicare in una Ls.

Per poter parlare di competenza grammaticale, riteniamo sia utile definirla.

\section{La Competenza Grammaticale}

Secondo le prime definizioni (Lado 1961, Carroll 1968) la competenza grammaticale consta di forme morfosintattiche che lo studente è capace di utilizzare nella comunicazione in una LS. Canale e Swain (1980) definiscono competenza grammaticale la conoscenza delle regole della fonologia, del lessico, della sintassi e della semantica, proponendo un modello di competenza grammaticale che include le caratteristiche lessico-grammaticali e semantico-grammaticali della lingua. Sulla base di questo modello, Bachman e Palmer (1996), descrivendo la conoscenza della lingua, definiscono la conoscenza grammaticale come conoscenza del vocabolario, della sintassi e della fonologia/ortografia a livello di sintagmi e di frasi, laddove la conoscenza testuale include la conoscenza della coesione e dell'organizzazione di una conversazione a livello discorsivo.

Pichiassi (1999: 212) sostiene che la competenza grammaticale si riflette nella conoscenza delle caratteristiche grammaticali e delle strutture di una Ls, ma anche nella capacità del loro uso. In altre parole, la competenza grammaticale è la capacità degli studenti di comprendere ed esprimere il significato attraverso la produzione e il riconoscimento delle frasi corrette, e non la capacità di memorizzare e ripetere le regole. Una definizione simile viene offerta da Brugè (2005: 42) secondo la quale la competenza grammaticale rappresenta la conoscenza dei principi e delle norme che regolano il livello di suoni, parole, frasi e significati. Tale competenza si manifesta nella capacità degli studenti di creare frasi che appartengono alla grammatica della lingua che viene appresa.

Per Larsen-Freeman (1991) la conoscenza grammaticale comprende tre dimensioni: forma linguistica, significato semantico e uso pragmatico. La forma linguistica comprende morfologia e modelli sintattici e riguarda principalmente l'accuratezza. La dimensione del significato descrive il significato trasmesso dalle strutture lessico-grammaticali, e riguarda la significatività dell'enunciato. L'uso si riferisce alle scelte lessicali e grammaticali che lo studente compie per conseguire una corretta comunicazione in un dato contesto. L'uso pragmatico descrive come e quando una forma linguistica viene utilizzata in un dato contesto, al posto di altre forme, soprattutto se entrambe trasmettono significati identici o simili. Purpura (2004: 59) ritiene che la dimensione dell'uso sia la più difficile da misurare nei test grammaticali 
perché non possiamo sapere se lo studente usa una forma perché la ritiene più adatta al contesto, o perché questa è l'unica forma che lui conosce.

Tutti gli autori citati sottolineano che la grammatica comprende la dimensione della forma e del significato. Questo è molto importante nella fase della verifica perché, secondo Purpura (2004: 81), il test grammaticale non dovrebbe verificare la conoscenza delle regole, bensì il loro utilizzo effettivo in un determinato contesto, sia a livello di frase che di testo.

In materia di prove grammaticali, è importante stabilire se vogliamo verificare la conoscenza esplicita o quella implicita della grammatica, o entrambe.

\section{La Conoscenza Grammaticale Implicita ed Esplicita}

Una delle questioni strettamente connesse all'insegnamento della grammatica è se la lingua si apprende meglio adottando la forma di insegnamento esplicita o implicita. Per rispondere a questa domanda, è necessario chiarire i concetti di conoscenza implicita ed esplicita della lingua. Questi due termini sono nati nell'ambito delle ricerche della psicologia sperimentale, e sono entrati nella teoria di acquisizione delle lingue grazie a Krashen (De Graaff, Housen, 2009: 732) secondo cui i processi impliciti sono legati all'acquisizione, laddove quelli espliciti all'apprendimento di una Ls.

La conoscenza grammaticale implicita (Ellis 2003: 105) viene dimostrata attraverso la produzione scritta e orale dell'apprendente, e non include la capacità dello studente di spiegare le regole grammaticali. Lo studente è quasi inconsapevole di ciò che studia (DeKeyser 2003: 314). Per Hulstijn (2002: 193) l'apprendimento implicito come attività inconscia e non pianificata richiede la costante esposizione ad una Ls. Per questo motivo, nel contesto di insegnamento della lingua italiana come Ls piuttosto che L2 che caratterizza il sistema scolastico montenegrino, è difficile parlare di conoscenza implicita della grammatica.

La conoscenza esplicita è la conoscenza consapevole delle forme grammaticali e del loro significato, ossia la capacità degli studenti di formulare le regole dell'uso delle strutture grammaticali, e di solito comporta l'uso di un metalinguaggio (Doughty, Williams, 2009a: 232). L'apprendimento esplicito è un processo più consapevole e più controllato, ed è quindi più suscettibile all'insegnamento rispetto all'apprendimento implicito.

L'insegnamento implicito è un modello in cui lo studente viene esposto alle strutture grammaticali e ai significati, prevedendo che poi le usi nella comunicazione, senza che l'insegnante gli fornisca alcuna spiegazione sul funzionamento del sistema linguistico. L'istruzione esplicita, al contrario, comprende spiegazioni relative alle strutture linguistiche e alle regole per il loro uso nella produzione in una Ls.

I modelli di insegnamento implicito della grammatica si basano principalmente sui principi relativi all'acquisizione della grammatica della L1: il linguaggio viene acquisito attraverso l'esposizione degli studenti alla Ls, la comunicazione con gli insegnanti o con altri studenti, senza alcuna attività cosciente rivolta all'insegnamento della grammatica. Krashen (1981, 1982, 1985), il principale sostenitore dell'insegnamento implicito, sottolinea che la grammatica (nonché la lingua in generale), si impara meglio se allo studente viene fornito un input linguistico adeguato, piuttosto che attraverso la spiegazione, gli esercizi o la correzione degli errori grammaticali.

L'apprendimento e l'acquisizione del linguaggio sono due processi diversi: l'acquisizione rappresenta un'interiorizzazione naturale delle forme linguistiche e dei significati attraverso l'esposizione alla Ls, mentre l'apprendimento si basa sulla comprensione e sull'applicazione consapevole delle regole che lo studente riceve dall'insegnante 0 dal libro di testo. Krashen ritiene che non ci sia nessun trasferimento dall'apprendimento all'acquisizione, ossia che le regole apprese attraverso le spiegazioni esplicite, non possono mai portare alla conoscenza implicita di una lingua straniera (Pichiassi, 1999: 170).

Bisogna, però, dire che numerosi studi condotti in ambienti dove gli studenti acquisicono una L2 e non la Ls indicano la mancanza di insegnamento implicito, dimostrando cioè che l'esposizione alla lingua target di per sé non è sufficiente per raggiungere alti livelli di competenza comunicativa nella Ls (Doughty, Williams, 2009: 2). In questo senso, si può concludere che, nel contesto formale dell'insegnamento delle lingue straniere, è necessaria una componente esplicita nell'insegnamento comunicativo o nell'insegnamento basato sui task, perché si tratta dell'unico modo per raggiungere i livelli di accuratezza grammaticale desiderati (Ur, 2011: 511, De Graaff, Housen 2009: 733).

Abbiamo già sottolineato che l'obiettivo dell'insegnamento della grammatica dovrebbe essere l'abilitazione degli studenti alla produzione linguistica in cui l'apprendente utilizzerà le strutture grammaticali correttamente, ossia lo sviluppo della conoscenza implicita (Ur, 2011: 507). La questione fondamentale è se I' insegnamento esplicito contribuisce al raggiungimento di questo obiettivo, e se la conoscenza implicita e quella esplicita sono in qualche modo collegate.

La relazione tra la conoscenza implicita ed esplicita della grammatica può essere espressa in tre modi: il legame tra queste due conoscenze non esiste, c'è una correlazione debole oppure vi è una forte correlazione tra questi due tipi di 
conoscenza (De Graaff e Housen 2009: 734).

Krashen sottolinea che tra la conoscenza implicita ed esplicita non c'è alcun collegamento e che le conoscenze esplicite non verranno mai interiorizzate nel sistema implicito di conoscenze linguistiche. La conoscenza implicita non può essere sviluppata attraverso l'insegnamento e la conoscenza esplicita acquisita attraverso l'istruzione esplicita gioca un ruolo rrilevante nell'uso di una lingua straniera e nello sviluppo della competenza comunicativa.

L'ipotesi di una debole correlazione tra questi due tipi di conoscenza, li vede come due sistemi separati. L'insegnamento della grammatica non può influire direttamente sulla conoscenza implicita e le conoscenze esplicite non possono trasferirsi in quelle implicite, sebbene possano aiutare l'apprendente a sviluppare la sua conoscenza implicita permettendogli di notare le differenze tra le strutture presenti nell'input e le strutture che utilizza nella produzione linguistica in una LS. II trasferimento dalla conoscenza esplicita a quella implicita dipende dalla capacità e dalla volontà degli studenti di integrare la nuova struttura grammaticale nel proprio sistema linguistico (De Graaff, Housen, 2009: 734; Ur, 2011: 512).

II ruolo della conoscenza esplicita nel processo di acquisizione di una lingua straniera, viene rappresentato da Ellis (1997: 123). L'input può essere acquisito, ossia può diventare intake soltanto se lo studente nota una nuova struttura grammaticale. Solo in seguito la forma linguistica diventa parte della sua interlingua se l'apprendente riesce - attraverso un processo di confronto - a notare le differenze rispetto all'interlingua attuale. In caso di singole parole o formule brevi (il cosiddetto item learning), l'apprendimento può avvenire anche in modo implicito perché non ci sono grossi problemi. Se si tratta di strutture complesse o di sistemi di regole (system learning), il processo diventa più complesso. In questo caso, la conoscenza esplicita acquisita attraverso la focalizzazione sulla forma può aiutare lo sviluppo della conoscenza implicita.

DeKeyser (2009: 56) sostiene che certamente può esserci, anche spesso, un trasferimento diretto dalla conoscenza esplicita a quella implicita. Tale trasferimento si ottiene attraverso gli esercizi. Gli studenti nel corso delle lezioni iniziano prima a sviluppare la conoscenza esplicita della lingua (attraverso la presentazione esplicita di regole ed esercizi grammaticali), per poi elaborarla e automatizzarla (attraverso esercizi comunicativi) fino a quando la conoscenza esplicita si trasformerà in quella implicita (De Graaff e Housen, 2009: 734).

Ellis (2008: 880-881) riporta i risultati di studi che indicano chiaramente che gli studenti che sono stati esposti a una forma di istruzione esplicita, nel corso dei test dimostrano migliori risultati rispetto agli studenti che non hanno ottenuto nessuna spiegazione relativa all'uso delle strutture grammaticali, o che non hanno ricevuto un feedback correttivo. Questa informazione è particolarmente importante nel contesto di apprendimento della Ls e non della L2, dove l'unico contatto con la lingua straniera avviene due o tre volte a settimana in aula.

Gli studi di cui sopra sostengono l'ipotesi che la riflessione grammaticale e gli esercizi grammaticali comunicativi facilitano il trasferimento della conoscenza esplicita delle regole in conoscenza implicita, ossia permettono l'uso automatico del linguaggio.

\section{Verifica della Competenza Grammaticale}

Abbiamo già indicato che la grammatica comprende la dimensione della forma e del significato e che può essere verificata a livello di frase e di discorso. Purpura (2004: 81) fa notare che è difficile verificare in modo separato le componenti della conoscenza grammaticale, perché esse sono strettamente correlate nell'uso del linguaggio, così che la maggior parte delle prove misura contemporaneamente più componenti. Tuttavia, è opportuno specificare le componenti della grammatica che stiamo cercando di misurare. In ogni caso, il test grammaticale non dovrebbe verificare la conoscenza delle regole ma il loro utilizzo effettivo.

Dimitrijevic (1999: 135) ritiene che lo scopo delle prove grammaticali è quello di determinare il grado in cui gli studenti possono manipolare i modelli sintattici a livello ricettivo e produttivo, se possono capire, riconoscere e produrre una frase grammaticalmente corretta, composta da elementi che sono stati presentati e su cui si è esercitato, ossia, se è in grado di comprendere e utilizzare produttivamente quelli che potremmo definire i modelli sintattici di base che una particolare situazione richiede.

Se abbiamo stabilito che la verifica delle competenze grammaticali dovrebbe includere sia la forma che il significato nel contesto, si pone la questione della scelta delle strutture grammaticali che faranno parte del test. Harris (1969: 25) ci offre un semplice suggerimento: il test dovrebbe includere un elenco completo delle strutture che sono state presentate durante il corso, e ogni struttura nel test dovrebbe occupare tanto spazio quanto ne ha occupato in classe.

Dopo aver scelto le strutture grammaticali che faranno parte del test, bisogna decidere se vogliamo verificare la competenza grammaticale implicita oppure esplicita.

Con il test di grammatica esplicita si verifica la grammatica indipendentemente dalle altre abilità linguistiche (Rea- 
Dickins, 2001: 251). In queste prove si usano tecniche quali la scelta multipla, il completamento, l'individuazione degli errori, ecc. Queste attività sono utili perché aiutano a capire se gli studenti sono in grado di applicare determinate forme grammaticali in un contesto in cui non si richiede l'uso di un linguaggio spontaneo. Tuttavia, per poter stabilire se gli studenti hanno completamente acquisito le strutture grammaticali, e se sono in grado di utilizzarle automaticamente nella comunicazione spontanea, è opportuno includere nel test esercizi che verifichino le conoscenze implicite. Per questi test utilizzeremo prove di produzione orale o scritta in Ls. Non si tratta quindi di tipiche prove di verifica della competenza grammaticale, in quanto, oltre alla grammatica, coinvolgono altri tipi di competenze.

Nella nostra ricerca, abbiamo cercato di verificare sia la competenza grammaticale esplicita che quella implicita degli alunni delle scuole medie del Montenegro. Le strutture che abbiamo inserito nel test sono state scelte in base al sillabo per la scuola media in Montenegro.

Prima di tutto, è necessario sottolineare che la lingua italiana si studia nelle scuole medie del Montenegro come seconda lingua straniera. Si studia nel terzo ciclo, dal VII al IX anno di studi. Alla fine del percorso didattico, gli alunni dovrebbero conseguire un livello di conoscenza A2. Bisogna anche ricordare che, nel 2005, in Montenegro è stata introdotta una riforma del sistema scolastico in occasione della quale, dal sillabo di italiano, sono state eliminate alcune strutture, come ad esempio: il trapassato prossimo, il passato remoto, il condizionale passato, tutti i modi indefiniti e tutti i tempi del congiuntivo. Con la riforma si è cercato di adeguare il sillabo montenegrino a quelli utilizzati in Italia per il livello A2. II sillabo attuale ${ }^{1}$ per le scuole medie in Montenegro prevede le seguenti strutture grammaticali: nomi: genere e numero; pronomi: personali, possessivi, dimostrativi, indefiniti, complemento oggetto, complemento di termine; articolo: indeterminativo, determinativo, partitivo; aggettivi: qualificativi, possessivi, numerali, indefiniti, gradi di comparazione; verbi: indicativo presente, imperativo, indicativo passato prossimo, indicativo imperfetto, futuro semplice, condizionale presente; avverbi; preposizioni proprie, improprie e articolate; congiunzioni e particelle ci e ne.

Per realizzare il nostro obiettivo, ossia stabilire la competenza grammaticale degli alunni montenegrini che studiano l'italiano, abbiamo adoperato metodi qualitativi e quantitativi. Siamo partiti dall'ipotesi per cui nelle scuole del Montenegro prevale ancora un metodo tradizionale di insegnamento basato sulle conoscenze grammaticali esplicite. Per questa ragione, abbiamo supposto che i risultati del test avrebbero dimostrato una buona conoscenza esplicita e una scarsa conoscenza implicita della grammatica, ossia che gli alunni non sarebbero stati in grado di utilizzare le strutture apprese nella comunicazione scritta. Abbiamo altresì supposto che alcune strutture non sarebbero state acquisite dalla maggioranza degli alunni, dato che risulta che il sillabo delle scuole medie del Montenegro per il livello A2 è molto più ampio dei corrispondenti sillabi utilizzati nell'insegnamento dell'italiano Ls in Italia.

La ricerca è stata effettuata in 25 scuole medie di 11 città, per un totale di 547 alunni. Selezionando i risultati ottenuti, siamo riusciti a stabilire il livello base della competenza grammaticale per il livello A2.

II test comprendeva 5 esercizi. Nel primo esercizio era necessario completare il testo con le forme corrette degli aggettivi tra parentesi (5 domande) mentre nel secondo bisognava completare il testo con la parola opportuna fra le tre sottoelencate ( 10 domande). II terzo esercizio prevedeva il completamento di un testo con le forme del presente o del passato prossimo indicativo dei verbi tra parentesi (10 domande), mentre nel quarto esercizio gli alunni dovevano scegliere la forma giusta del verbo tra le tre proposte (15 domande).

L'ultimo esercizio prevedeva la composizione di un tema su un argomento che gli alunni avevano già avuto modo di trattare nel corso delle lezioni di italiano. Ciò al fine di stabilire sia le abilità ricettive che quelle produttive relative all'uso delle strutture grammaticali.

Ogni domanda nel test era destinata al controllo di una precisa struttura grammaticale in riferimento alla sua funzione. In base ai risultati del test, abbiamo stabilito quattro livelli di conoscenza delle strutture grammaticali. Le domande alle quali ha risposto meno del $25 \%$ degli alunni, ci hanno dimostrato che gli alunni non conoscono la struttura in questione, ossia che non sono in grado di usarla correttamente. Le domande alle quali ha risposto correttamente il 25$49,99 \%$ dei candidati, dimostrano che sono ancora presenti errori sistematici nell'utilizzo della struttura target. II dato in base a cui alla domanda ha risposto in modo corretto il 50-74,99\% dei candidati ci ha dimostrato che gli alunni, in linea di massima, sanno usare la struttura in questione, mentre la percentuale tra il 75 e il $100 \%$ indica che gli alunni conoscono benissimo la struttura grammaticale.

In base a questa suddivisione siamo riusciti a stabilire la competenza grammaticale degli alunni definendola in forma di descrittori grammaticali. Gli alunni del Montenegro, dopo tre anni di studio di lingua italiana nelle scuole medie:

non sanno usare:

- l'imperfetto per esprimere un'azione in corso in un determinato momento del passato (22,9\%)

1 Izborni predmeti. Predmetni programi: engleski, francuski, ruski, italijanski, njemački jezik VII, VIII i IX razred devetogodišnje osnovne škole, Podgorica, Zavod za školstvo, 2005. 
- il futuro semplice per esprimere insicurezza o dubbio (23\%);

in prevalenza non sanno usare:

- il condizionale presente per esprimere un dubbio o un consiglio $(28,5 \%)$

- il condizionale presente per esprimersi in modo cortese $(30,7 \%)$

- l'imperativo per esprimere esortazioni e ammonizioni (31,7\%)

- l'infinito dopo le locuzioni impersonali con il verbo essere $(34,8 \%)$

- le preposizioni improprie per indicare relazioni nel tempo e nello spazio $(37,7 \%)$

- le congiunzioni per spiegare causa, tempo, fine e sim. $(38,4 \%)$

- i pronomi interrogativi per formulare domande $(40,8 \%)$

- il plurale degli aggettivi qualificativi per descrivere persone, oggetti e sim. (44,6\%)

- i pronomi e gli aggettivi possessivi per indicare il possesso (45,7\%)

- il futuro semplice per esprimere azioni situate nel futuro $(47,5 \%)$

in linea di massima sanno usare:

- il passato prossimo dei verbi regolari e irregolari, dei verbi riflessivi per esprimere azioni completate nel passato $(50,3 \%)$

- l'infinito con i verbi modali e altri verbi come desiderare, preferire e sim. $(53,4 \%)$

- le forme impersonali dei verbi regolari e irregolari al presente $(53,4 \%)$

- le forme atone del pronome in funzione di complemento oggetto per esprimere la forma di cortesia $(54,1 \%)$

- il passato prossimo per indicare un'azione che interrompe un'altra azione in corso $(55,8 \%)$

- gli aggettivi qualificativi al singolare per descrivere persone, oggetti e sim. (60,1\%)

- l'articolo indeterminativo per introdurre nel discorso un nome di cui non si era parlato in precedenza $(61,8 \%)$

- l'indicativo presente dei verbi regolari e irregolari per esprimere e descrivere azioni in corso $(63,37 \%)$

sanno usare benissimo:

- verbi sapere e conoscere per indicare nomi di persone e città oppure la capacità di fare qualcosa $(78,2 \%)$

- forme del verbo al presente per distinguere forme familiari e forme di cortesia $(78,2 \%)$

- $\quad$ presente indicativo del verbo esserci per indicare la presenza di qualcuno o di qualcosa $(79,9 \%)$

- gli aggettivi dimostrativi per esprimere una relazione spaziale tra il parlante, l'oggetto del discorso e colui che ascolta (82,4\%)

- forme toniche di pronomi in funzione di complemento oggetto per esprimere particolare rilievo nella frase $(82,4 \%)$

- le preposizioni semplici IN e A con i nomi geografici $(83,9 \%)$

- il presente del verbo esserci per indicare la presenza o l'esistenza

- le forme del verbo per distinguere forme familiari e forme di cortesia

Come abbiamo accennato, l'ultimo esercizio era destinato a controllare se gli alunni fossero o meno in grado di applicare le conoscenze grammaticali nella produzione scritta, ossia abbiamo cercato di verificare le loro conoscenze grammaticali implicite. I risultati che abbiamo ottenuto non sono soddisfacenti. Abbiamo stabilito scarsa presenza e uso scorretto di: passato prossimo, futuro semplice, aggettivi, preposizioni, pronomi personali, avverbi, articoli, accordo di numero e genere tra nomi e aggettivi. L'unica struttura grammaticale di cui hanno mostrato una buona conoscenza è il presente indicativo. I risultati precisi li riportiamo qui di seguito: corretto di:

Nella produzione scritta degli alunni, quindi, abbiamo stabilito scarse conoscenze degli alunni in relazione all'uso

- articolo determinativo e indeterminativo e accordo di genere e numero tra articoli e nomi $(25,7 \%)$

- accordo di numero e genere tra nomi e aggettivi del sintagma nominale $(27,2 \%)$

- $\quad$ pronomi personali $(30,7 \%)$

- $\quad$ preposizioni per esprimere rapporti di spazio e tempo $(31,2 \%)$

- aggettivi, in primo luogo aggettivi possessivi e uso di articolo $(32,5 \%)$

- verbi ausiliari $(33,7 \%)$

- futuro semplice per esprimere azioni future o progetti $(36,1 \%)$

- passato prossimo per esprimere azioni avvenute nel passato $(39,5 \%)$

- $\quad$ avverbi (46,8\%).

Abbiamo stabilito che gli studenti possiedono buona conoscenza della:

- del presente indicativo per esprimere azioni in corso e azioni in futuro $(50,8 \%)$ 
Da quanto esposto in precedenza, possiamo concludere che gli studenti non possiedono un'adeguata conoscenza dell'uso e della formazione dell'imperfetto, né dell'uso del futuro per esprimere incertezza, supposizione. La ragione di questo si potrebbe ricercare nel libro di testo in uso che non prevede queste due strutture, e possiamo supporre che in certe classi queste strutture forse non siano state nemmeno presentate, qualora gli insegnanti abbiano seguito solo il percorso previsto dal manuale e non dal sillabo. Dall'altra parte, forse queste due strutture non dovrebbero nemmeno far parte del livello A2 dato che il sillabo CILS prevede che strutture quali futuro, condizionale, imperativo e imperfetto vengano introdotte a livello B1 (Benucci, 2007: 152). L'unica struttura che gli studenti sanno usare abbastanza bene, sia a livello ricettivo - produttivo che a livello produttivo, è il presente indicativo.

Ci scoraggiano i risultati dell'ultimo esercizio dal quale deduciamo che la maggior parte degli alunni non possiede buone conoscenze in relazione all'uso pragmatico delle strutture grammaticali. La spiegazione potrebbe essere quella che l'insegnamento si basa ancora sul metodo grammaticale - traduttivo e non sui metodi recenti che vedono l'insegnamento della grammatica strettamente correlato ad altre abilità linguistiche.

In conclusione possiamo stabilire che le nostre ipotesi di partenza erano corrette, ossia che gli studenti non conseguono il livello A2 nell'uso pragmatico delle strutture grammaticali e che, nella comunicazione libera, non sono in grado di utilizzare gran parte delle strutture grammaticali previste dal sillabo. In altre parole, gli alunni dopo la scuola media possiedono un livello medio di competenza grammaticale esplicita e un livello basso di competenza grammaticale implicita. Per risolvere questo grave problema, ossia per abilitare gli alunni alla produzione spontanea in lingua italiana, riteniamo sia utile adoperare nuovi metodi di insegnamento della grammatica (processazione dell'input, insegnamento mediante l'uso di task e simili). Presentare le strutture grammaticali in modo induttivo all'interno di esercizi che promuovono la comunicazione in lingua italiana, aiuterebbe di sicuro gli alunni a trasferire almeno una parte delle conoscenze esplicite nelle conoscenze implicite che consentono l'uso abbastanza automatico della lingua.

\section{Bibliografia}

Bachman, L. F., Palmer A. S. (1996). Language Testing in Practice. Oxford: OUP.

Barki, P. et al. (2003). Valutare e certificare l'italiano di stranieri. I liveli iniziali. Perugia: Guerra edizioni.

Benucci, A. (1994). La grammatica nell'insegnamento dell'italiano a stranieri. Roma: Bonacci editore.

Benucci, A. (2007). Sillabo di italiano per stranieri. Una proposta del Centro Linguistico dell'Università per Stranieri di Siena. Perugia: Guerra edizioni.

Bojičić, G. (2014). La competenza grammaticale nell'insegnamento dell'italiano Ls - livello A2. (Atti della conferenza "L'talia e la cultura europea", tenutasi il 17.-18.10.2013 presso l'Università Jagellonica di Cracovia), in stampa.

Canale, M., Swain, M. (1980). Theoretical bases of communicative approaches to second language teaching and testing, in: Applied Linguistics, 1980, 1, pp. 1-47.

Carroll, J. B. (1968). The psychology of language testing. In A. Davies (ed.). Language Testing Symposium: A Psycholinguistic Approach, London: OUP, pp. 46-69.

De Graaff, R., Housen, A. (2009). Investigating the Effects of L2 Instruction. In C. J. Doughty, M. H. Long (ed.). The Handbook of Language Teaching. West Sussex: Blackwell, pp. 726-755.

DeKeyser, R. (2003). Implicit and explicit learning. In C. Doughty, M. Long (eds.). Handbook of Second Language Acquisition. Oxford: Blackwell, pp. 313-348.

Dekeyser, R. (2009). Beyond focus on form: Cognitive perspectives on learning and practising second language grammar. In C. Doughty and J. Williams (ed.). Focus on form in classroom second language acquisition. Cambridge: Cambridge University Press, pp. 42-63.

Dimitrijević, N. (1999). Testiranje u nastavi stranih jezika. Beograd: Zavod za udžbenike i nastavna sredstva.

Doughty, C., Williams, J. (2009). Focus on form in Classroom Second Language Acqusition. Cambridge: Cambridge University Press.

Doughty, C., Williams, J. (2009a). Pedagogical choices in focus on form. In C. Doughty, J. Williams (ed.). Focus on form in Classroom Second language Acquisition. Cambridge: Cambridge University Press, pp. 197-261.

Ellis, R. (2003). Task-based Language Learning and Teaching. Oxford: OUP.

Ellis, R. (2008). The study of second language acquisition. Oxford: OUP.

Harris, D. P. (1969). Testing English as a Second Language. New York: McGraw-Hill.

Hulstijn, J. H. (2002). Towards a unified account of the representation, processing and acquisition of second language knowledge, in:Second Language Research, 18, pp. 193-223.

Izborni predmeti. Predmetni programi: engleski, francuski, ruski, italijanski, njemački jezik VII, VIII i IX razred devetogodišnje osnovne škole. (2005). Podgorica: Zavod za školstvo.

Krashen, S. (1981). Second language acquisition and second language learning. Oxford: OUP.

Krashen, S. (1982). Principle and Practice in Second Language Acquisition. Oxford: Pergamon Press.

Krashen, S. (1985). The Input Hypothesis: Issues and Implications. New York: Longman.

Lado, R. (1961). Language Testing. New York: McGraw-Hill.

Larsen - Freeman, D. (1991). Teaching grammar. In M. Celce- Murcia. (ed.), Teaching English as a Second or Foreign Language. 
Boston, MA: Heinle and Heinle Publishers, pp. 279-296.

Pichiassi, M. (1999). Fondamenti di Glottodidattica. Temi e problemi della didattica linguistica. Perugia: Guerra edizioni.

Purpura, J. E. (2004). Assessing grammar. Cambridge: Cambridge University Press.

Rea-Dickins, P. (2001). Fossilization or evolution: the case of grammar testing, in M. Celce-Murcia (ed.). Experimenting with Uncertainty: Essays in Honour of Alan Davies, Cambridge: Cambridge University Press, pp. 251-263.

Ur, P. (1988). Grammar Practice Activities. A pratical guide for teachers. Cambridge: Cambridge University Press. 